\title{
Discussions on the Processing of the Multi-Component Seismic Vector Field
}

\author{
Chao Wang ${ }^{1}$, Yun Wang ${ }^{1, *}$, Pengyuan Sun ${ }^{2}$ and Yuanfang Li ${ }^{1}$ \\ 1 The State Key Laboratory of Ore Deposit Geochemistry, Institute of Geochemistry, Chinese Academy of \\ Sciences, Guiyang 550081, China; wangchao@mail.gyig.ac.cn (C.W.); liyuanfang18@mails.ucas.ac.cn (Y.L.) \\ 2 BGP INC. China National Petroleum Cor., Zhuozhou 072751, China; sunpengyuan@cnpc.com.cn \\ * Correspondence: yunwang@mail.iggcas.ac.cn; Tel.: +86-15985148520
}

Received: 12 March 2019; Accepted: 25 April 2019; Published: 28 April 2019

\begin{abstract}
Multi-component seismic data contain a great deal of vector field information that reflects the situation of the underground medium. However, the processing methods used for multi-component seismic data are still being developed, and effectively retaining and using this information is the difficulty and the focus of the task. Currently, the main-stream processing techniques of multi-component seismic data treat the individual components independently as a scalar field; in this way, they do not excavate the vector features of the wavefield, thus restricting the potential utilities of the effective information. Research into processing methods that are suitable for use with the vector field, which can better retain and use the orientations and the relative amplitude relationship between multi-component seismic data, is urgently needed and represent an important direction for the current development of multi-component seismic data processing techniques. In this paper, we introduce and summarize several existing vector pre-processing techniques, including polarization filtering, de-noising using vector order statistics, group sparse representation, and vector separation of compressional waves and shear waves, to help scholars develop more effective vector field processing methods and to promote the development of vector processing techniques for multi-component seismic data.
\end{abstract}

Keywords: Multicomponent seismic; Vector field; Polarization filtering; Multivariate order statistic filtering; Vector field separating

\section{Introduction}

The three-component seismic data in a three-dimensional Descartes system simultaneously record the linear motion of the particle in the vertical and horizontal directions as well as retain a relatively complete record of seismic wave translations. They not only simultaneously provide the travel time (velocity), amplitude, and frequency information of the compressional (P) and shear (S) waves but also provide the relative amplitude relationship of the same wave among different components, as well as the amplitude, frequency, phase, and travel time differences for different waves. The information of these vector wavefields would provide a more detailed and accurate characterization of the structure, lithology, fluid saturation, pore pressure, and fractures of an underground medium [1]; however, because of the limitations of current methods and processing techniques of seismic data, such information cannot be fully obtained. The advantages of the current multi-wave and multi-component seismic techniques are limited to the following [2-5]: (1) inversions of travelling time and waveform for the P-wave only [6,7]; (2) shear-wave birefringence analysis [8]; (3) compensation of a defect caused by the difficulty of imaging with a PP-wave under a gas cloud, a salt dune, or in a low-acoustic impedance area using PS-wave imaging [9,10]; (4) anisotropy and fracture identification [11]; and (5) prediction of reservoir lithology and fluids [12]. In particular, except for the prediction of the fast and slow 
shear polarization and the fracture azimuth that uses the vector field information of the S-wave, other methods and applications only use the scalar wavefield information of the P-wave and S-wave. If we were able to fully use the vector field information of the P-wave and S-wave, we would be able to more accurately estimate the anisotropic parameters [13], quantitatively describe the parameters of fractures and pores, and finely depict the reservoir lithology, or even directly identify oil and gas reservoirs. Wang et al. provided a very detailed discussion of the significance of studies on the geophysical vector field [14].

The prerequisite for making the best use of the vector information of a seismic wavefield is that the processing of the seismic data must retain the vector characteristics of the wavefield. Even the ordinary processing of amplitude compensation and de-noising should be performed very carefully; the original scalar field method cannot simply be adopted $[15,16]$ to independently process each component. Otherwise, the processing is prone to interrupt the relative amplitude relationship of the vector data and cause mistakes in subsequent applications of the vector information. Therefore, the processing system of multi-wave and multi-component seismic data not only should be a simple expansion of a single-component processing system of the P-wave but also should possess a set of processing methods and procedures that are specifically suitable for the multi-component vector field. So far, some studies have treated the multi-component seismic data as a vector field. For example, Zhao et al. [17] and Kamath et al. [18] applied the full-waveform inversion of a multi-component seismic elastic wave to estimate the elastic parameters of the shear modulus, Lame constant, and anisotropy. The vector wavefield separation method proposed by Zhang et al. [19] and Wang et al. [20] can retain the vector form of the P-wave and S-wave data after their separation with no distortion in the amplitude and phase. The reverse time migration imaging technology of the elastic wave proposed by $\mathrm{He}$ et al. [21] and Li et al. [22] directly used the vector field as the input. However, these vector processing techniques are still in the initial stages of research, and they are only sporadically distributed on a small number of independent links of the processing procedure for multi-component seismic data. It is difficult to effectively exhibit the holographability (containing abundant dynamics and kinematics information) of a vector field and the superiority of a vector processing method.

In this paper, we introduce existing vector de-noising techniques for multi-component seismic data. The earliest vector de-noising technique for multi-component seismic data was polarization filtering. It was first applied to studies of natural earthquakes. For example, Vidale [23] proposed a method of three-component instantaneous polarization property analysis and applied this method to effectively identify the seismic phase and arrival time of the SV-wave, Love wave, and the Rayleigh wave in the recording of the San Fernando Earthquake in 1971. Crampin and Lovell [24] gave an overview of how to use the polarization vector of the fast and slow shear waves to detect anisotropy and crustal stress. Lilly and Park [25] proposed a method of time-frequency polarization analysis, which was used to separate and reconstruct various types of waves that interfere with each other in an earthquake recording. Thereafter, polarization filtering was expanded and applied to suppress surface-waves in multi-component seismic data, separate fast and slow S-waves, and de-noise random signals. Diverse methods were developed, including the time-domain [26], frequency-domain [27], wavelet transform domain [28], and t-f-k domain [29] methods. Several filtering methods based on vector order statistics, which are widely applied to color image processing, are very suitable for processing vector signals. Wang et al. [30] and Xun et al. [31] applied these methods to the random noise suppression of multi-component seismic data and achieved good results. In addition, the random noise attenuation method proposed by Rodriguez et al. [32], based on the $3 \mathrm{C}$ group sparsity constrained time-frequency transform, can process the multiple components simultaneously, and retain the relative amplitude relationship among the components and the weak effective signal well. Separation of the P-waves and S-waves is a special de-noising processing, which is very important in processing multi-component seismic data. The vector separation of P-waves and S-waves proposed by Lei [33], Lu et al. [34], and $\mathrm{Li}$ [35] can retain the intrinsic properties of a vector wavefield. 


\section{Multi-Component De-Noising Methods with Respect to the Vector Seismic Field}

\subsection{The Polarization Filtering Method}

Different types of seismic waves have different polarization manners during propagation. The surface wave exhibits elliptical polarization characteristics, the P-wave exhibits linear polarization characteristics in the direction of the wave propagation, while the S-wave exhibits linear polarization characteristics perpendicular to the direction of the wave propagation, which provides the theoretical basis for identifying and separating various wave types with their differences in polarization characteristics. Polarization filtering is usually used to separate the body and surface waves in the field of earthquake research $[36,37]$ and suppress random noise $[38,39]$, while in exploration seismology, it is mostly used for surface-wave suppression and seldom used for the attenuation of random noise.

The earliest method of polarization analysis occurred in the time domain [40]. Thereafter, Jurkevics [41] and Chen et al. [42] conducted detailed studies. The time-domain polarization analysis constructs a covariance matrix using the multi-component seismic data within a certain time window and computes the polarization property of the wave within this time window using the characteristic value and characteristic vector of the constructed covariance matrix. The difficulty with time-domain polarization filtering is the selection of the time-window length because different lengths result in different polarization parameters. Therefore, Diallo et al. [43] and Ma [44] proposed to adaptively select the length of the time window according to the instantaneous frequency of the signal; Rene et al. [45], Morozov and Smithson [46], Vidale [23], Schimmel and Gallart [47], and Lu et al. [26] proposed the method of Complex Trace Analysis (CTA) based on the Hilbert transform to obtain the instantaneous polarization properties of the multi-component seismic signals.

However, these methods of time-domain polarization analysis cannot differentiate the various wave types that are mutually superposed in time. Park et al. [48] and Reading et al. [27] proposed the frequency-domain polarization filtering method to differentiate the waves that are superposed in time but with different frequency components. However, the body waves and surface waves would be superposed in both time and frequency; therefore, polarization analysis methods based on a time-frequency transform were developed, including the continuous wavelet transform-based method [28,49], the discrete wavelet transform-based method [50], and the S transform-based methods [51,52]. To solve the problem of the inaccurate estimation of the polarization parameter caused by the phase distortion of the conventional wavelet transform coefficient, Galiana-Merino et al. [53] proposed to use the stationary wavelet packet transform to estimate the instantaneous polarization parameters.

Methods of polarization filtering in the time-frequency domain simultaneously use the travel time, frequency, and polarization features of seismic waves, but these methods belong to the single-channel processing mode. Another significant difference between the surface wave and body wave, namely, the apparent velocity, cannot be used. Therefore, Wang et al. [29] proposed to develop a complex seismic data gathering technique by taking the radial component as the real part and the vertical component as the imaginary part, before applying the polarization analysis in the $\mathrm{t}-\mathrm{f}-\mathrm{k}-$ domain for surface-wave attenuation. If the surface wave and body wave are different in either time, frequency, or apparent velocity, the surface waves can be automatically identified and eliminated according to the polarization rate in the $\mathrm{t}-\mathrm{f}-\mathrm{k}$ domain, which fully reflects the advantages of the vector processing method. An example is given here to exhibit the effectiveness of the $t-f-k$ domain polarization filtering method. The synthetic two-component seismic data are shown in Figure 1 where Figure $1 \mathrm{a}$ is the vertical component, and Figure $1 \mathrm{~b}$ is the radial component. The dominant frequency is $30 \mathrm{~Hz}$ for P-waves and $22 \mathrm{~Hz}$ for PS-waves. Considering that the frequency range of the ground roll is $5-20 \mathrm{~Hz}$, it shows considerable overlap between the frequency of the PS-waves and the ground roll. The most challenging issue is that the apparent velocities of the ground roll and the reflected wave in far offset are very close. A comparison of the $\mathrm{t}-\mathrm{f}-\mathrm{k}$ domain polarization filtering with the f-k filtering and time-frequency domain polarization filtering would convince that the $t-f-k$ domain polarization filtering performs more effectively. As shown in Figure 1c,d, the results of f-k filtering work well but 
not perfectly. The ground roll is not completely removed from the filtered result. Additionally, some body waves appear in the removed noise profiles, as shown in Figure 1e,f; this is because the apparent velocity of these body waves is close to the apparent velocity of the ground roll. Figure 2a,b show the filtered results of the time-frequency polarization filtering. Because the body wave and ground roll overlap both in the time domain and frequency domain, they cannot be effectively separated in the time-frequency domain. The $\mathrm{t}-\mathrm{f}-\mathrm{k}$ polarization filtering is implemented in the $\mathrm{t}-\mathrm{f}-\mathrm{k}$ domain. Three $\mathrm{f}-\mathrm{k}$ slices (from time 150, 650, and $950 \mathrm{~ms}$ ) of the $\mathrm{t}-\mathrm{f}-\mathrm{k}$ transform of the gathered complex seismic data are shown in Figure 3a,c,e. These figures show a meaningful phenomenon; the body waves have equal energy while the ground roll has discrepant energy in the positive and negative frequency planes. The ground roll allocated more energy in the positive frequency plane. The f-k slices taken from different times are different from each other; therefore, the computed filtering windows should change with time, as shown in Figure $3 b$,d,f. With the automatically identified filtering windows, the filtered results of the $\mathrm{t}-\mathrm{f}-\mathrm{k}$ polarization filtering are shown in Figure 4a,b. Furthermore, the removed ground roll is shown in Figure 4c,d. The test results indicate that the $\mathrm{t}-\mathrm{f}-\mathrm{k}$ polarization filtering method can suppress the ground roll effectively and guarantee the body wave does not drop out.
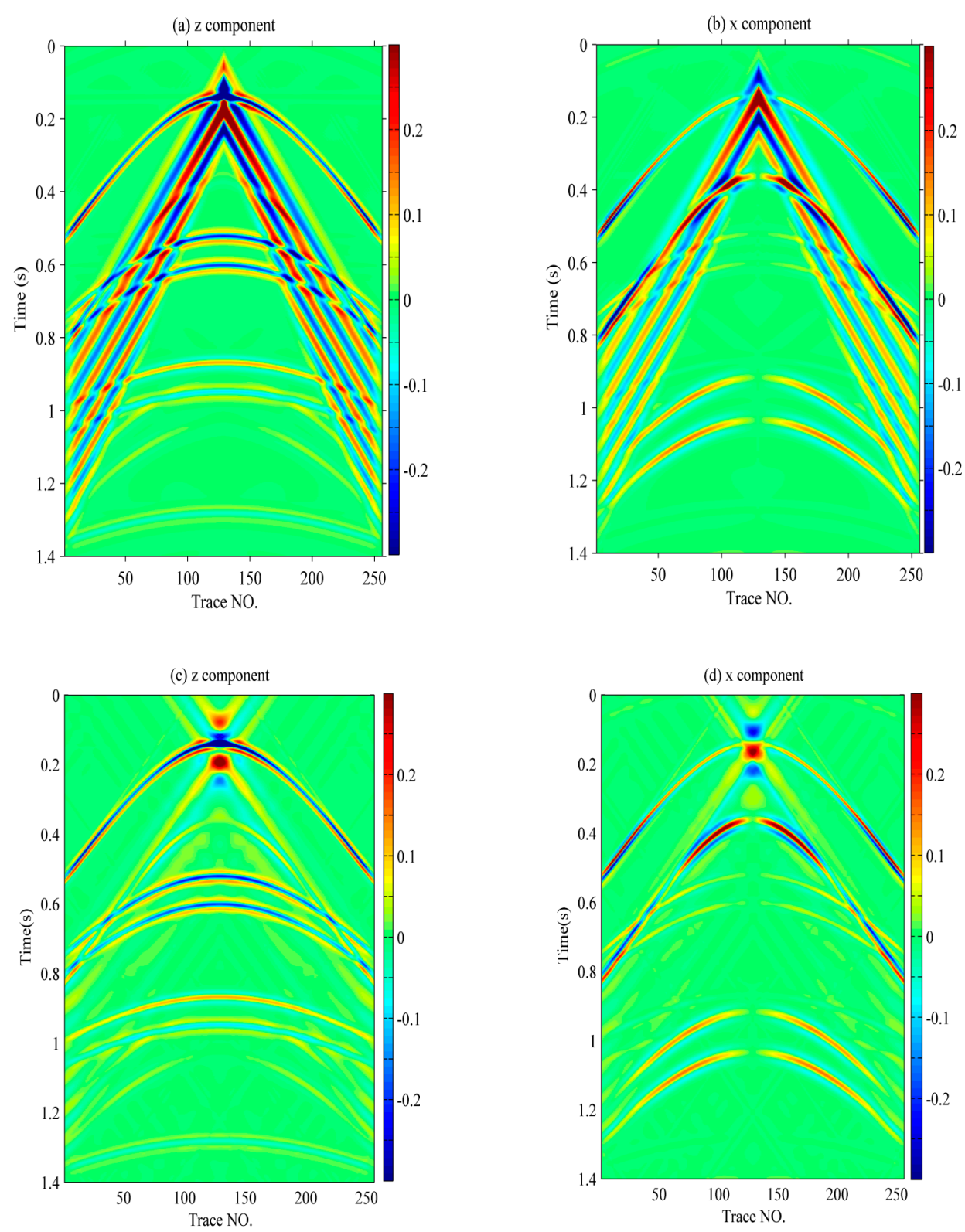

Figure 1. Cont. 

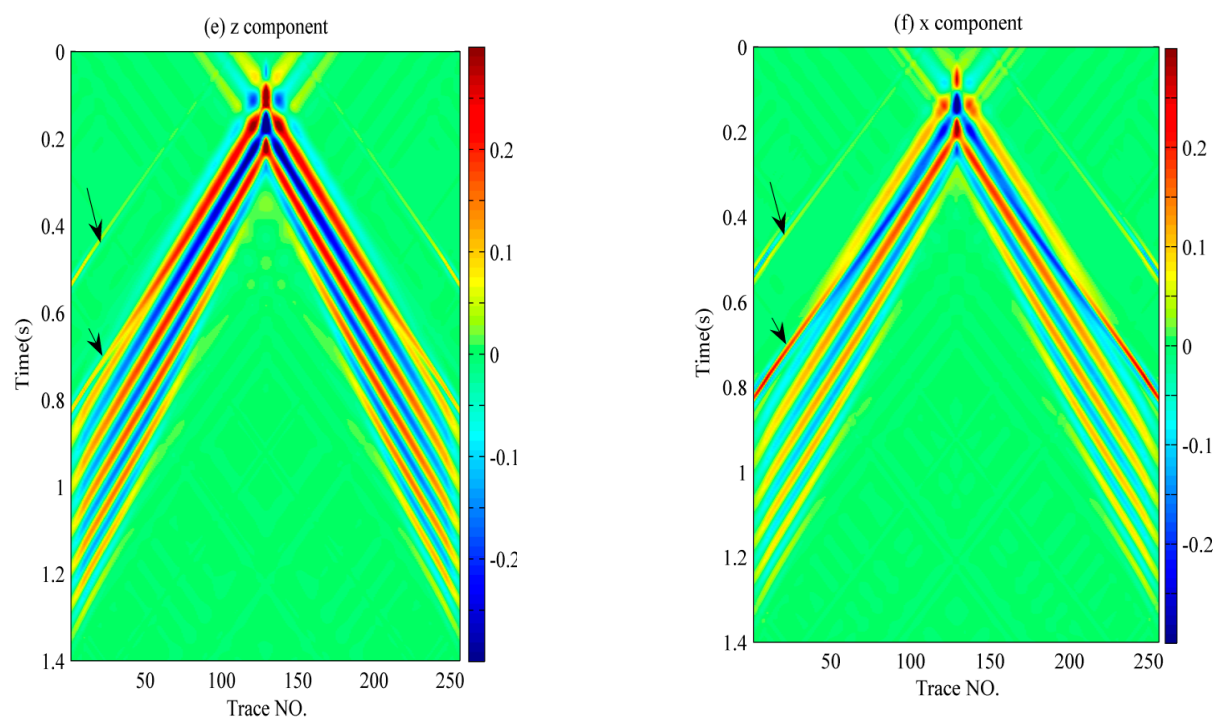

Figure 1. Synthetic two-component shot gathered data. (a) Vertical component, (b) radial component, (c) filtered vertical component, and (d) radial component with f-k filtering. The removed noise from the (e) vertical component and (f) radial component.
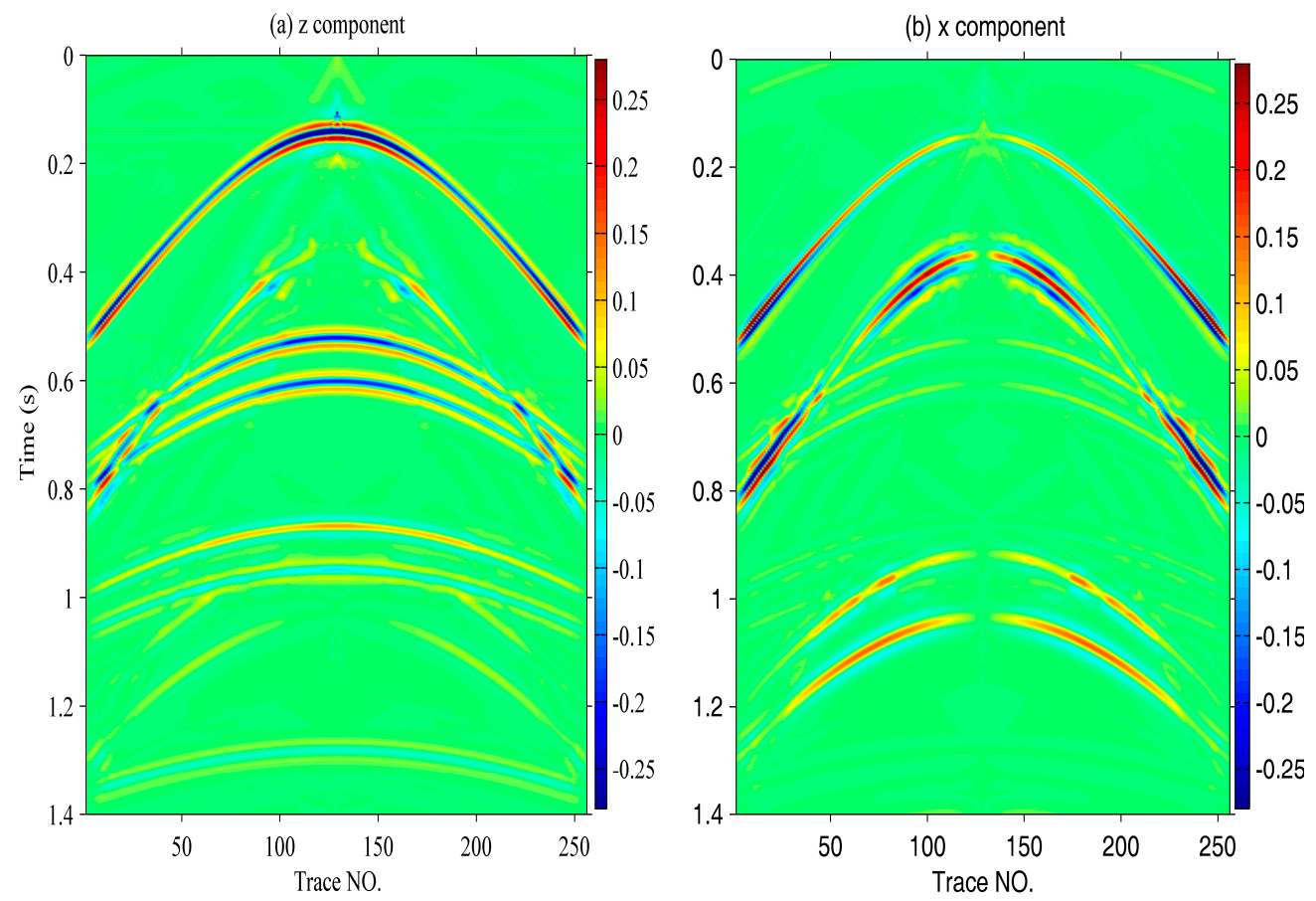

Figure 2. (a) Filtered vertical component (b) and radial component with time-frequency polarization filtering. 

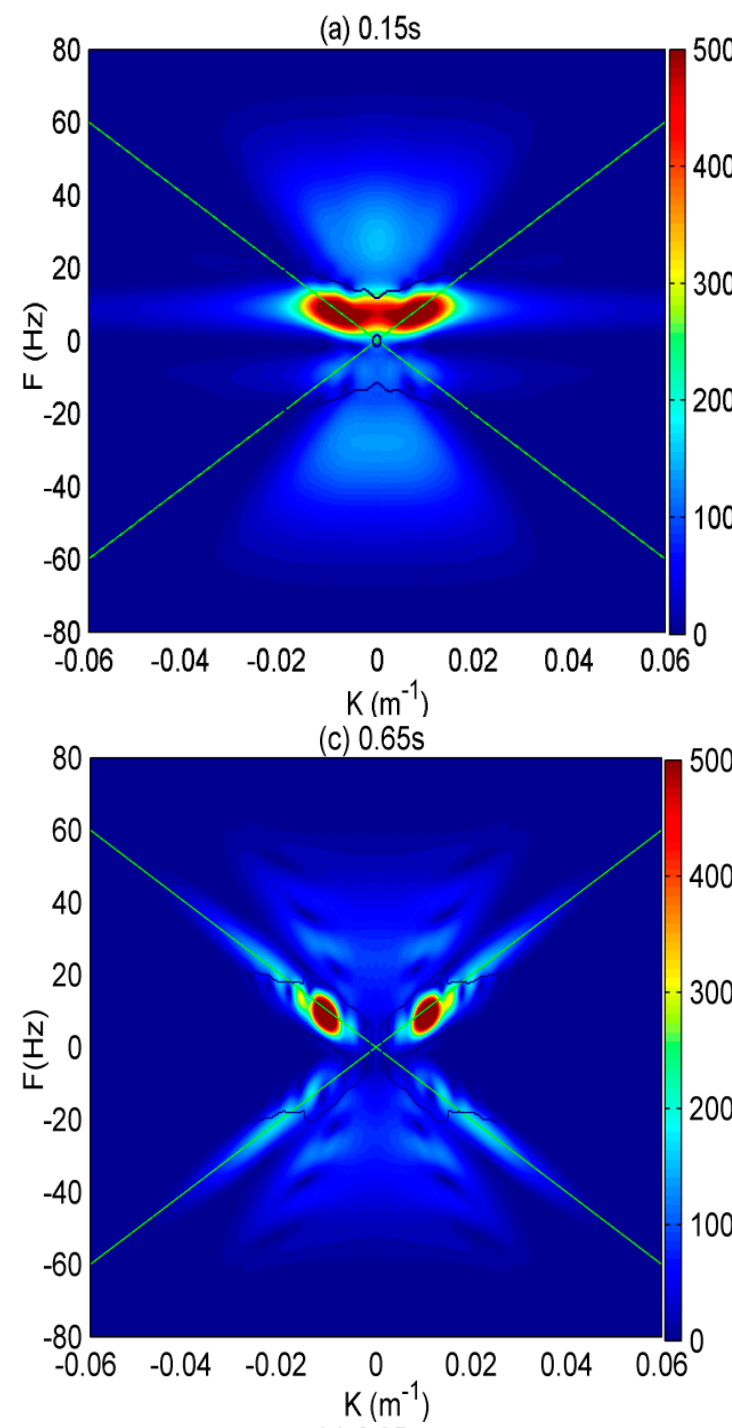

(e) $0.95 \mathrm{~s}$

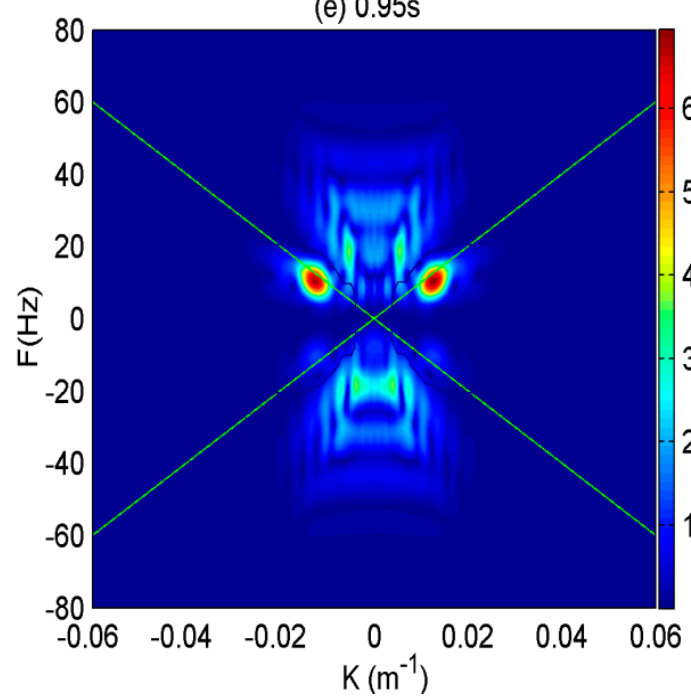

(b) $0.15 \mathrm{~s}$

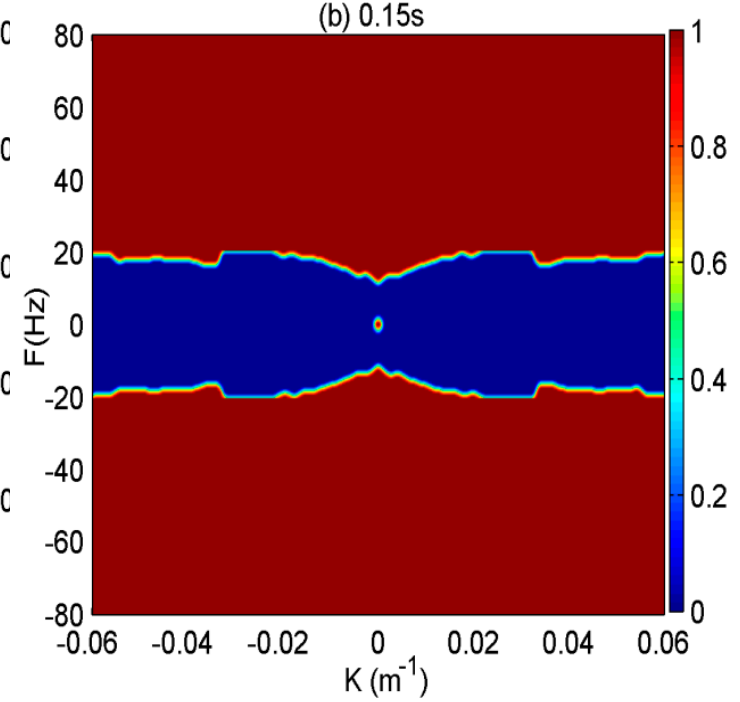

(d) $0.65 \mathrm{~s}$
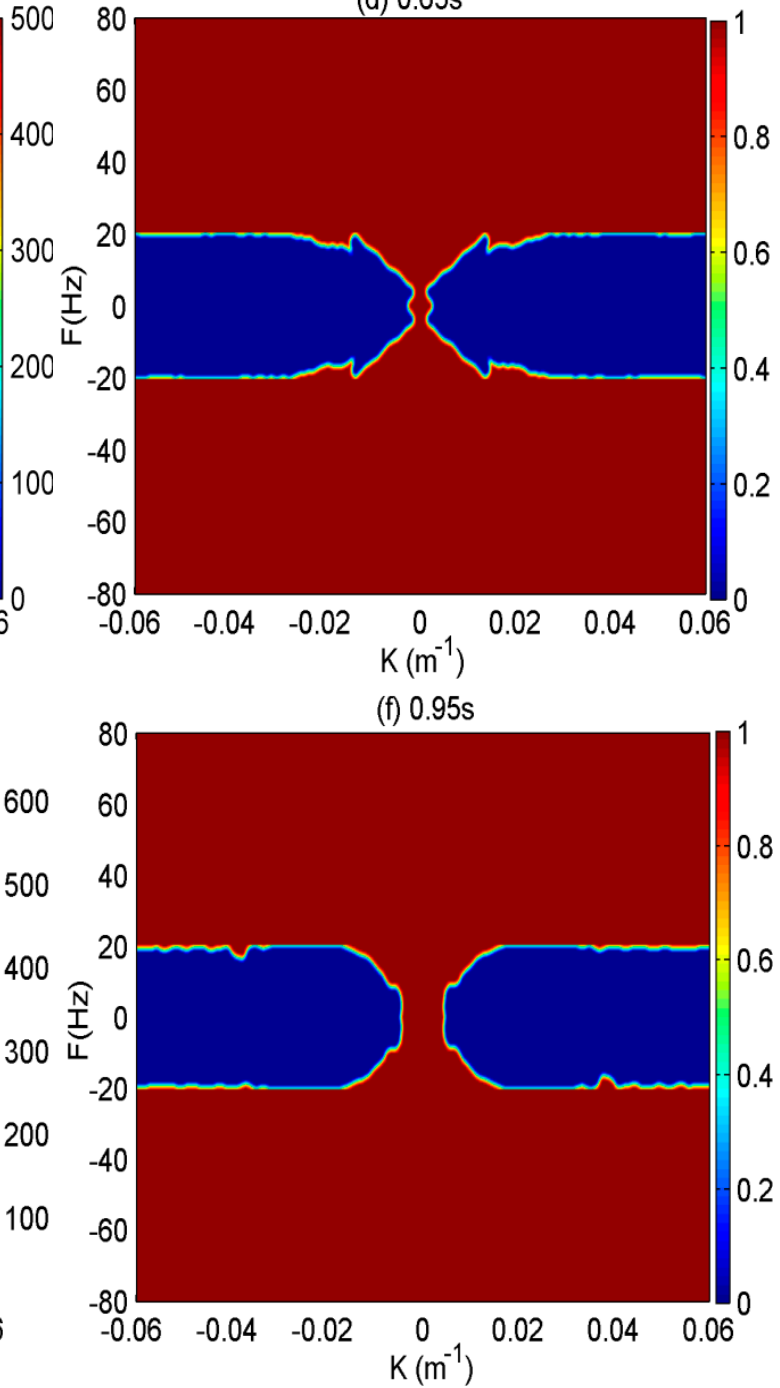

Figure 3. The f-k slices of the t-f-k transform taken from (a) $150 \mathrm{~ms}$, (c) $650 \mathrm{~ms}$, and (e) $950 \mathrm{~ms}$. The computed filtering windows at (b) $150 \mathrm{~ms}$, (d) $650 \mathrm{~ms}$, and (f) $950 \mathrm{~ms}$. 
(a) z component

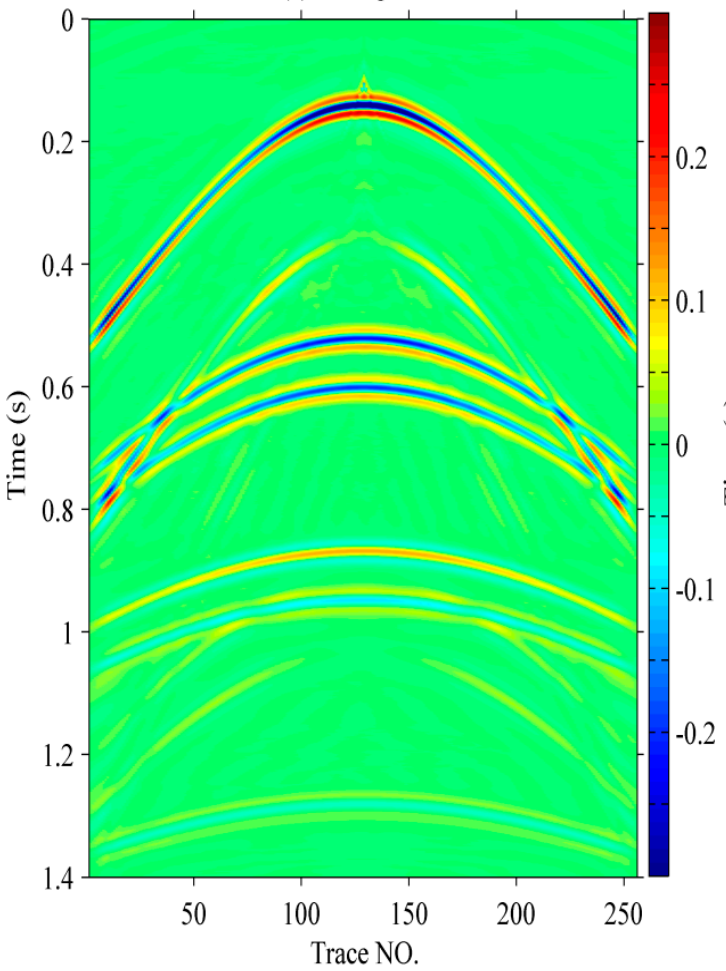

(c) z component

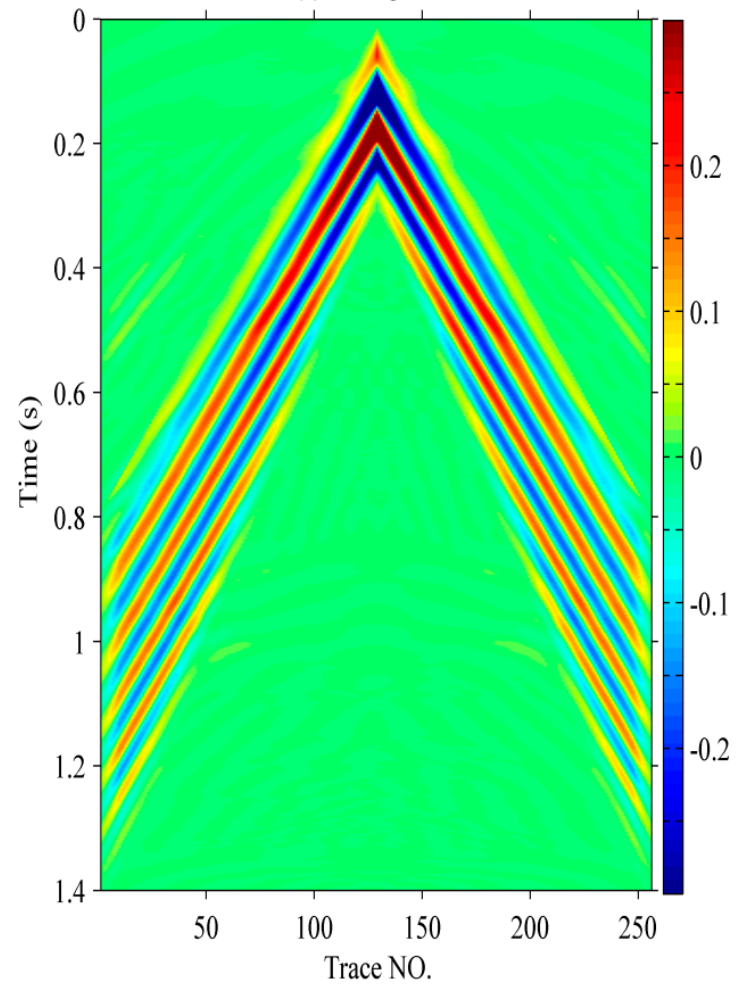

(b) $\mathrm{x}$ component

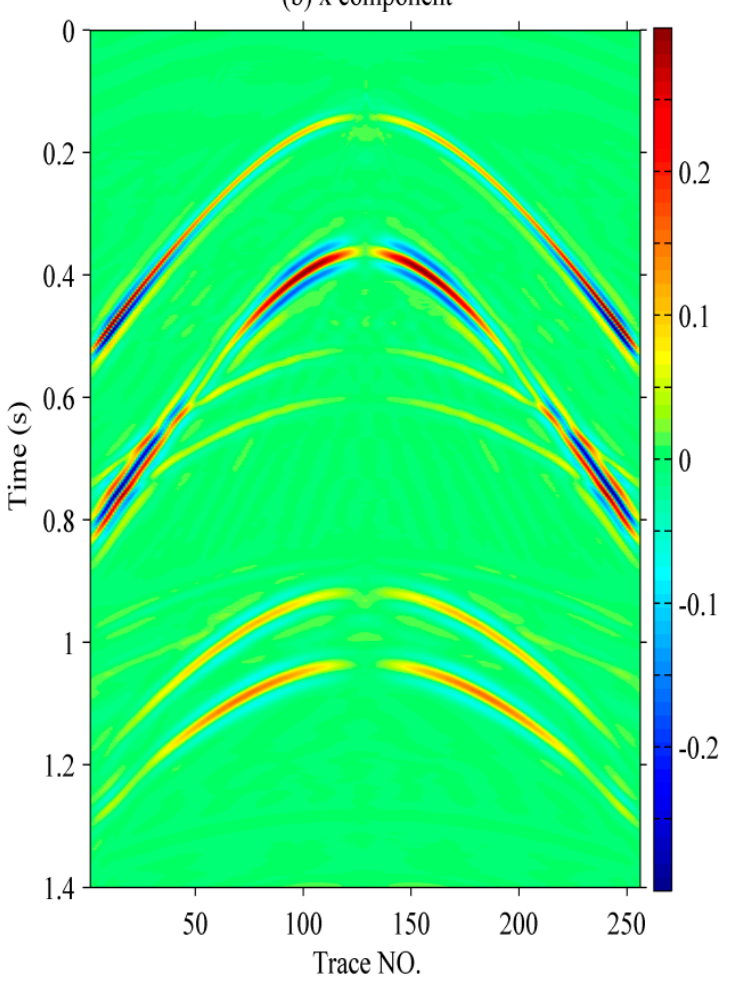

(d) $\mathrm{x}$ component

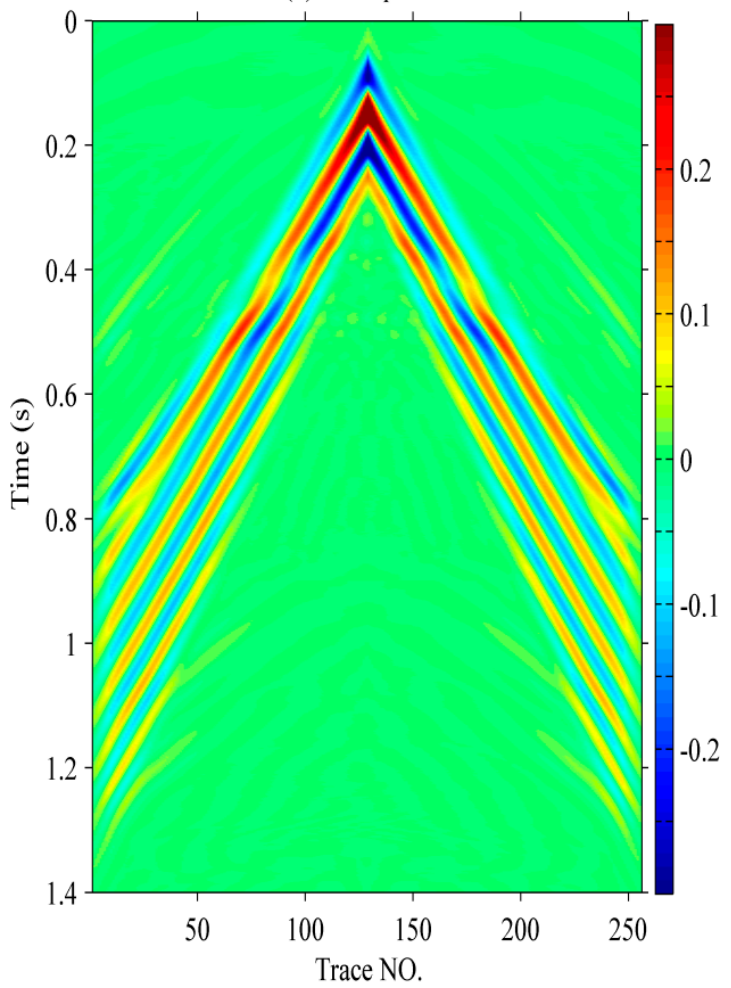

Figure 4. (a) The filtered vertical component (b) and radial component with the $t-f-k$ domain polarization filtering method, and (c) the removed noise from vertical component and (d) the radial component. 


\subsection{The Vector Wavefield Filtering Method Based on Vector Order Statistics}

Many studies have revealed that multivariate order statistic filters perform better in color image processing because they treat the color image as vector data whereas other filtering methods treat the color image as scalar data [54]. These multivariate order statistic filters can be classified into several categories according to the ordering method for multivariate data, such as the multi-channel $\alpha$-trimmed mean filter, multichannel Modified-Trimmed Mean (MTM) filter [55], Vector Median Filter (VMF) [56,57], and vector direction filter [58]. A study by Pitas et al. [59] indicated that the VMF is more applicable to suppressing noise that has a long-tail distribution, such as negative index noise and salt-and-pepper noise; the MTM filter is more suitable for suppressing noise that has a short-tail distribution, such as Gaussian white noise.

In recent years, Huo et al. [60] applied the VMF to seismic data processing and separated blended seismic data; Liu [61] used the VMF to eliminate direction anomalies of the local dip vectors of seismic events. In these two applications, however, the filter still processes every single component of the seismic data, and the vector is formed from the mathematical construction of some sampling points of adjacent traces or adjacent time, rather than a vector in physical space. Wang et al. [30] and Xun et al. [31] applied an extension of the vector order statistics-based filter to multi-component seismic data. First, according to the correlation between the vector signals of adjacent traces, the optimum local direction of the event was examined, and then, the vector points along the event were selected for vector order statistics-based filtering. Because every sampling point of a three-component detector records a vector, it is very intuitive, and there are practical advantages to adopting the vector order statistics-based filter to process three-component seismic data. Tests of the model data and real data showed that the noise-suppressing result using vector order statistics-based filtering is better than when using the signal-component approach [30]. The solid lines in Figure 5 show some hodograms of two-component seismic signal segments taken from the de-noised results with different methods, and the dashed lines are the hodogram curves of the uncontaminated seismic data. The first, second, and third columns are the results of low-pass filtering, single-component filtering, and vector order statistics-based filtering, respectively. From top to bottom, there are four seismic signal segments which are taken from different detectors and different events. We can see that vector order statistics-based filtering maintains the vector information of multicomponent seismic data more effectively than the single-component filtering. 

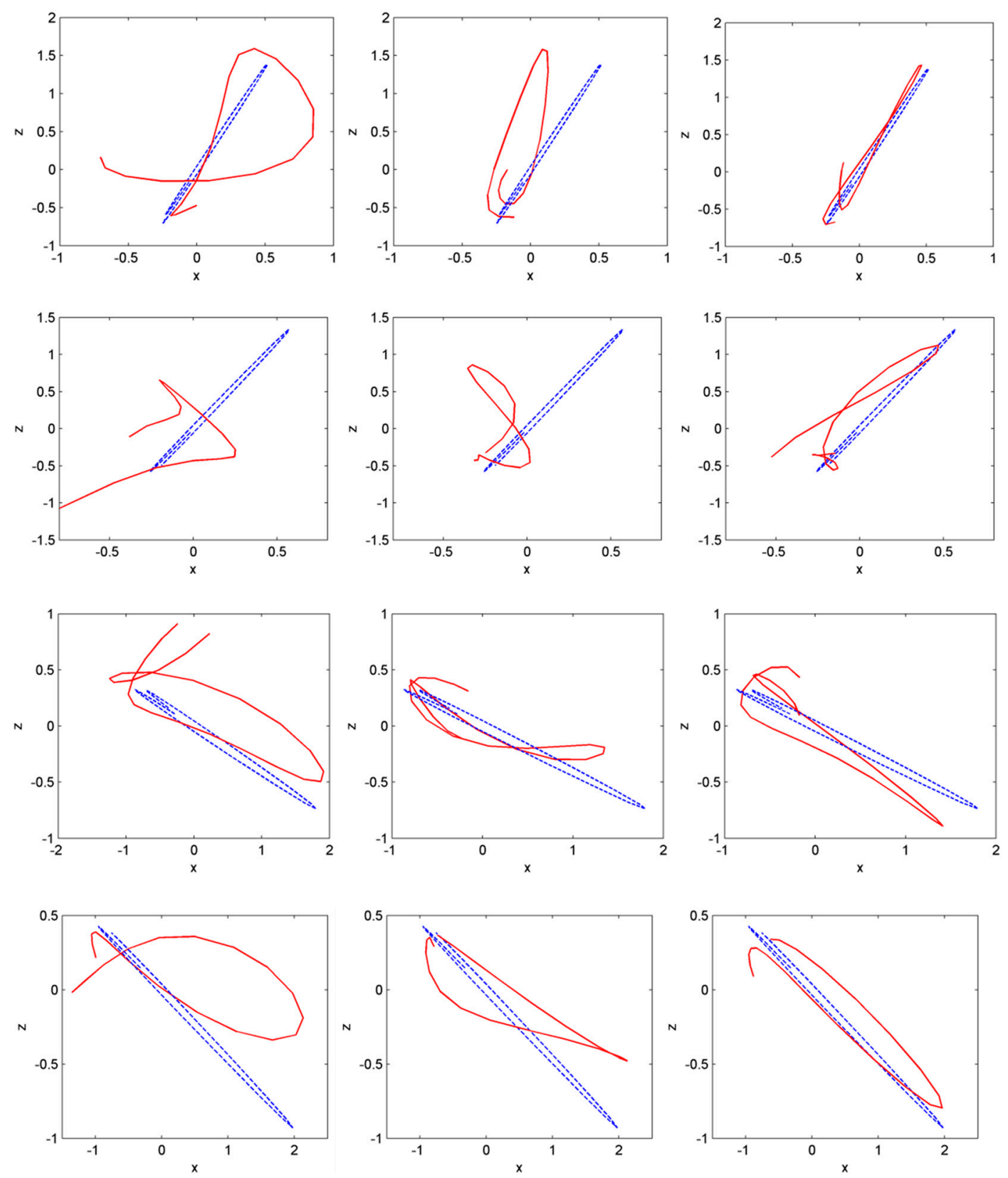

Figure 5. Hodograms of two-component seismic signal segments, dashed lines are uncontaminated seismic data, and solid lines are the de-noised results using different methods. The first, second, and third columns are the results of low-pass filtering, single-component filtering, and vector order statistics-based filtering, respectively.

\subsection{Multi-Component Joint De-Noising Based on the Group Sparsity Representation}

Expressing the time-domain noise-containing signals in other domains through mathematical transforms is the approach often used in de-noising seismic data and wavefield reconstruction. The mathematical transforms often used include Fourier transform, $\mathrm{S}$ transform, wavelet transform, and curvelet transform. These signal representation methods based on specific mathematical transformations can effectively represent the signal of a corresponding structural model according to the structural characteristics of the individual transform dictionaries. However, real seismic signals are very complex; therefore, it is difficult to achieve the sparse representation of seismic signals by only selecting a single transform method. Many overlaps in the representation coefficients of the noise and the effective signal still exist when using a single transform method. We combine the dictionary of 
multiple transforms to compose an over-complete dictionary or construct a new dictionary according to the signal characteristics. Through searching, we can only use a small number of elements in the over-complete dictionary for the sparse representation of complex signals [62,63]. Because many fast and effective algorithms of sparse representation have been proposed in recent years, such as the matching pursuit algorithm [64], the orthogonal matching pursuit algorithm [65], and the base tracking algorithm [66], sparse representation has been widely applied in seismic data processing. Applications include the reconstruction of the wavefield [67], surface-wave attenuation [68,69], and de-convolution [70]. To retain the relative amplitude relationship among the different components of multi-component seismic data after de-noising, Rodriguez et al. [32] developed and applied the time-frequency transform based on the group sparsity constrained representation to de-noise three-component micro-seismic data. They believe that because the micro-seismic events recorded by the three-component detector have the same arrival time and frequency component for the three components, the coefficient of sparse representation for the effective signal of the three components in the time-frequency domain should have the same distribution mode. In other words, the coefficients of the three components at the same time-frequency point are either all zero or nonzero. With the coefficients of three-component data at the same time-frequency point as one group, we can use the concept of group sparsity proposed by Yuan and Lin [71], Fornasier and Rauhut [72], and Eldar [73] to process the multicomponent seismic data. The three components $\mathrm{x}, \mathrm{y}$, and $\mathrm{z}$ can form an $\mathrm{N} \times 3$ matrix (where $\mathrm{N}$ is the number of time sampling points):

$$
\mathbf{S}=\left[\begin{array}{lll}
\mathbf{x} & \mathbf{y} & \mathbf{z}
\end{array}\right]
$$

The group sparsity constrained representation in an over-complete dictionary $\boldsymbol{\Phi}$ can be expressed as the following mathematical optimization problem:

$$
\hat{\mathbf{A}}=\operatorname{argmin}_{A}\left[\|\mathbf{S}-\boldsymbol{\Phi} \mathbf{A}\|_{2,2}^{2}+\lambda\|\mathbf{A}\|_{2,1}\right],
$$

where $\hat{\mathbf{A}}$ is the coefficient of sparse representation, and each column corresponds to a component. The symbol $\|\bullet\|_{p, q}$ represents the mod of the matrix, which is defined as

$$
\|\mathbf{X}\|_{p, q}=\left(\sum_{n}\left(\sum_{k}\left|x_{n, k}\right|^{p}\right)^{\frac{q}{p}}\right)^{\frac{1}{q}} .
$$

The optimization problem of Equation (2) can be solved by adopting the fast iterative shrinkage-threshold algorithm (FISTA) proposed by Beck and Teboulle [74]. While constructing the dictionary, Rodriguez et al. used the complex Ricker wavelet as the mother wavelet. The complex wavelet dictionary not only improves the sparsity of the representation coefficients but also retains the consistency between the representation coefficients for waves with different phases.

We applied the multi-component joint de-noising method based on the group sparsity constrained representation to synthesized seismic data. Figure 6a shows the three components of the synthesized data, and Figure $6 \mathrm{~b}$ shows the data contaminated with Gaussian white noise. The first waveform in the y component is completely submerged in the noise. We used the multi-component joint de-noising method based on the group sparsity constrained representation and the single-component de-noising method based on the sparse representation, separately, for the de-noising processing. The results are shown in Figure $6 \mathrm{c}$,d. We can see that the multi-component joint de-noising method can effectively attenuate random noise, and it can preserve weak signals more effectively than the single-component de-noising method. Beyond that, the multi-component joint de-noising method has incomparable advantage in reserving the vector information of the multi-component seismic signal, which is useful for successive processing. 
(a)

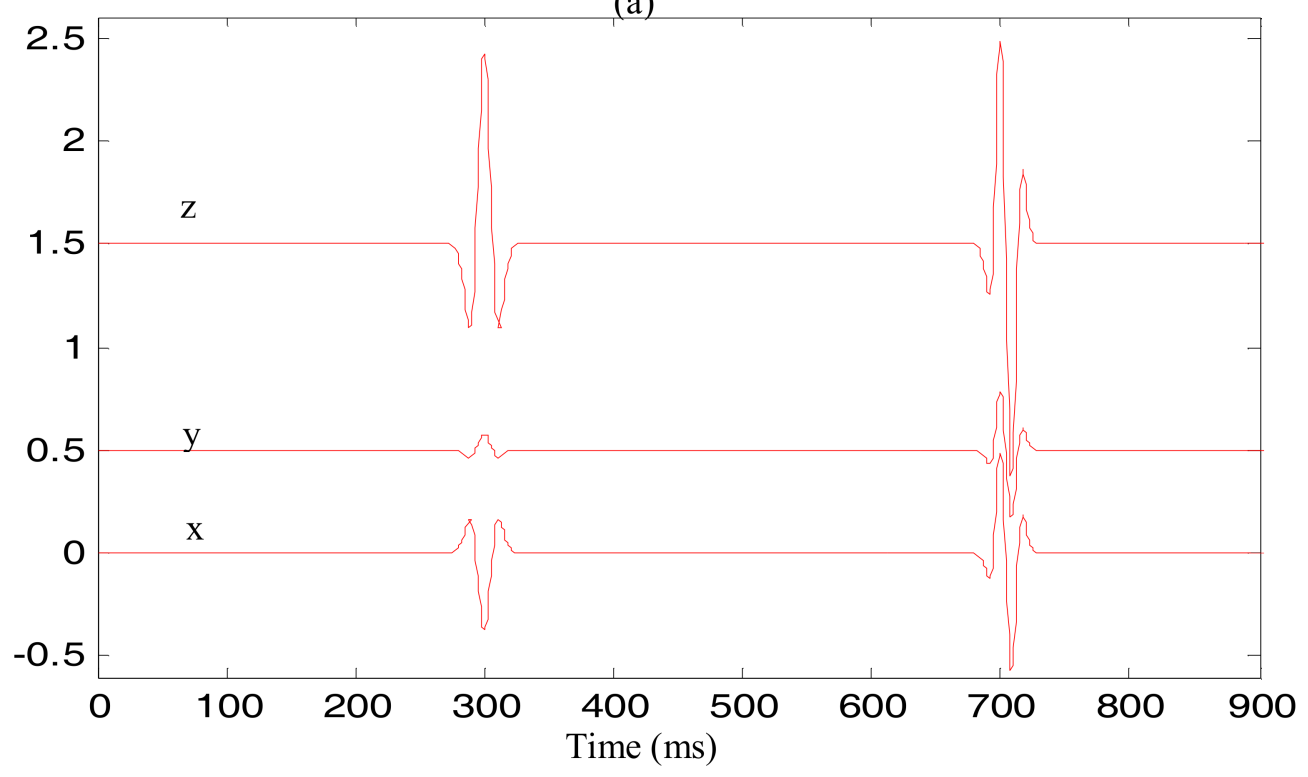

(b)

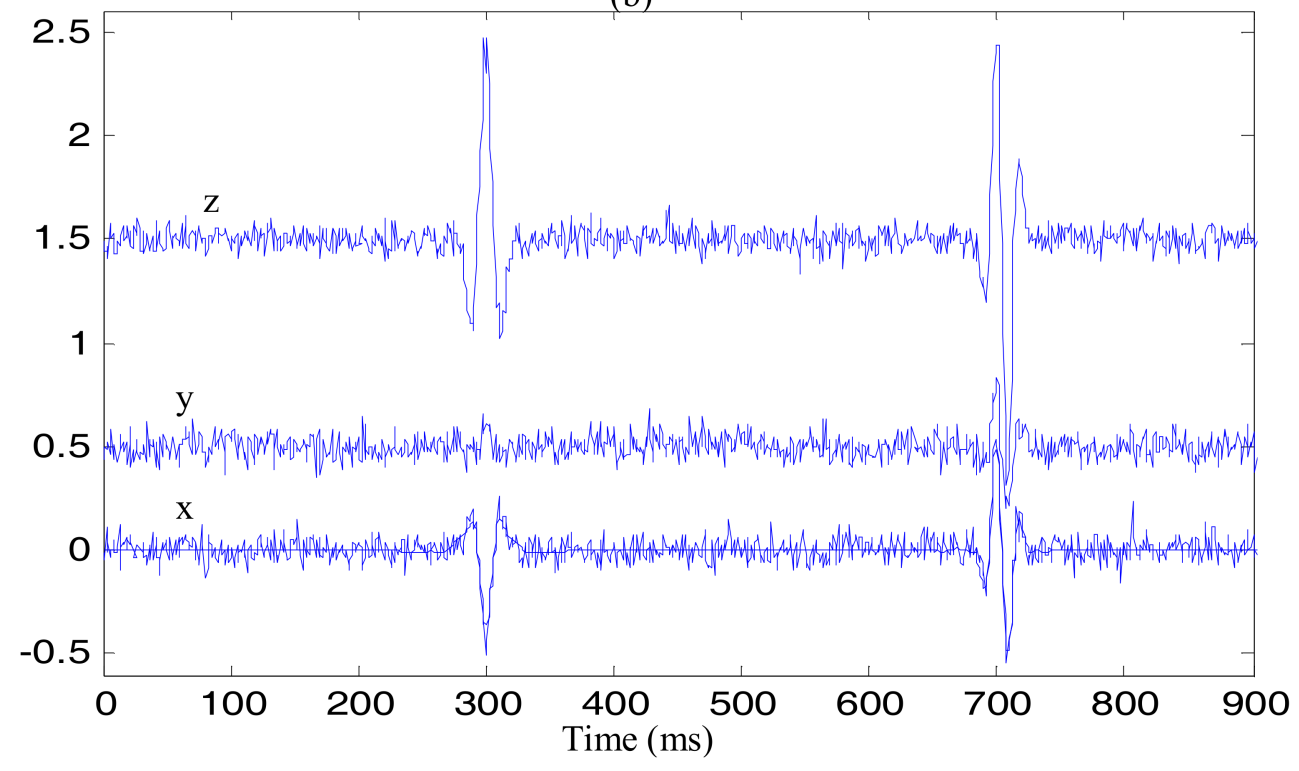

Figure 6. Cont. 
(c)

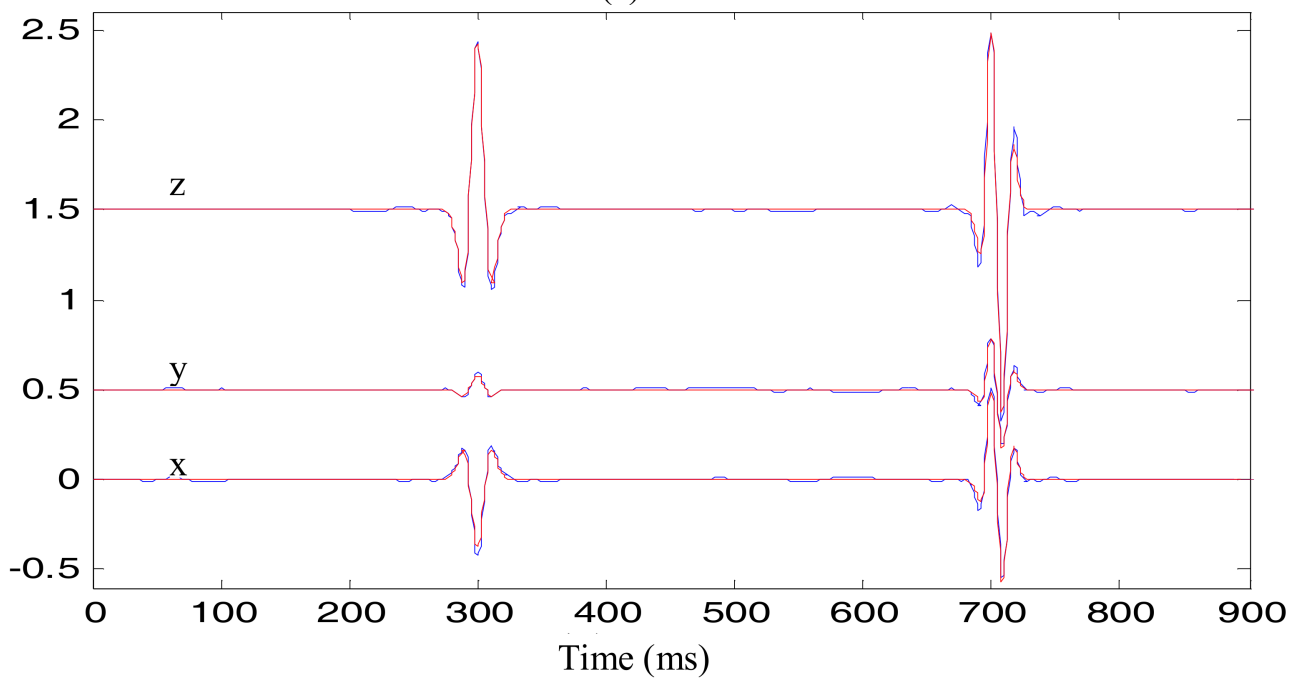

(d)

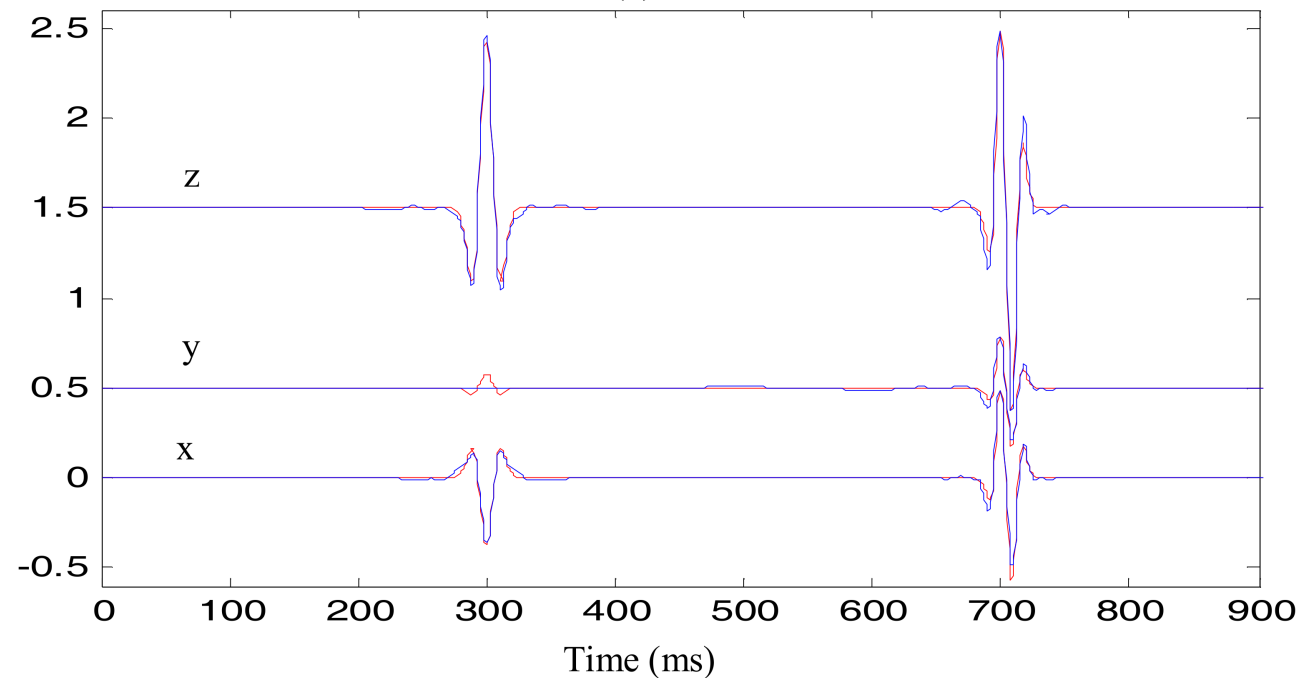

Figure 6. (a) Original seismic data, (b) seismic data contaminated with random noise, (c) de-noised data using the group sparsity constrained time-frequency transform (blue line), and (d) de-noised data using the sparsity constrained time-frequency transform (blue line).

\subsection{The Vector Separating Method of the P-wave and S-wave}

The separation of the P-wave and S-wave cannot only be used as a special de-noising method to eliminate the mutual interference between the P-wave and S-wave, but also as an important tool for the extraction of the pure wave and the reconstruction of the wavefield. For the conventional and scalar processing systems, separation of the P-wave and S-wave is the foundational step, while for the vector processing methods, separation of the P-wave and S-wave can reduce the complexity of the vector field data, which is favorable for the analysis of fast and slow S-waves and can increase the imaging quality. It should be emphasized that the subsequent multi-wave joint application requires the separated P-wave and S-wave, though the data must retain the original vector characteristics.

The wavefield separation method based on the Radon transform independently processes the single-component seismic data, and cannot retain the vector characteristics of multi-component seismic data [75]. Hu et al. [76] separated P-waves and S-waves according to the polarization direction of multi-component seismic data, but the separated P-waves and S-waves retrograded to the scalar field. Yao et al. [77], Sun et al. [78], and Yan and Sava [79] proposed a series of separation methods for P-waves and S-waves based on the divergence and curl of different media with homogeneous, 
inhomogeneous, and isotropic properties, but the separated P-waves became scalars. Moreover, the divergence and curl operation causes distortions in the phase and amplitude for the wavefield after separation; therefore, the results need to be corrected.

To ensure, after separation, that the P-waves and S-waves still retain the relative vector characteristics as in the original input data, $\mathrm{Li}$ [35] proposed a wave equation method for the separation of P-waves and S-waves in the frequency-wavenumber domain, which first separated the P-wavefield and S-wavefield from the total wavefield using the divergence and curl operation according to the Helmholtz decomposition principle. Thereafter, the method projected two wavefields in the vertical and horizontal directions by multiplying them with the normalized wave numbers in the vertical and horizontal directions. This method barely changes the phase and amplitude information of the $\mathrm{P}$-waves and S-waves. The derived $\mathrm{P}$-waves and $\mathrm{S}$-waves are still two components of $\mathrm{x}$ and $\mathrm{z}$ and retain the vector information of the original wavefield. However, this method is only applicable to the homogeneous isotropic surface medium with known P-velocity and S-velocity. For an inhomogeneous isotropic medium, Wang et al. [20] proposed a vector separation method of P-waves and S-waves based on the wavefield extrapolation. In this method, the P- and S-wave-separated elastic equation was first used to downward extrapolate the elastic wavefield from the surface to a reference depth, and the P-waves and S-waves were separated in the process of downward extrapolation. Only the P-waves were saved at the reference depth; then, the elastic wave equation was used to upward extrapolate the saved P-wave to the surface to obtain the separated P-wave recording. The P-waves were subtracted from the original recording of the total wavefield to obtain the separated S-wave recording. Li et al. [80] proposed a similar vector separation method for P-waves and S-waves, but they first used the elastic wave equation to downward extrapolate the elastic wavefield from the surface to a reference depth. Thereafter, they used the P- and S-wave-separated elastic equation to extrapolate the wavefield upward to the ground surface to obtain the separated P-waves and S-waves. Therefore, the P-waves and S-waves are separated naturally during the upward extrapolation, and their phase and amplitude are the same as before separation. The P- and S-wave-separated elastic equation used by these two vector separation methods is the equivalent form of the conventional coupled elastic wave equation proposed by Ma and Zhu [81], and it is only applicable to an isotropic medium. Using the vector decomposition method for an elastic wavefield developed by Zhang and McMechan [19], the vector separation method for P-waves and S-waves could be extended to an anisotropic medium.

The abovementioned vector wavefield separation methods all imply that the P-waves and S-waves are orthogonally polarized, and this assumption is generally not valid for an anisotropic medium [82]. For the wavefield separation problem of non-orthogonal P-waves and S-waves in an anisotropic medium, Lei [33] and Lu et al. [34] discussed this problem from the perspective of natural earthquakes and exploration seismology and provided a solution. The basic idea of the solution is to apply a rotational transform to transform the vertical and horizontal components based on the Cartesian coordinate system to the affine coordinate system composed of the wave vector directions of realistic P-waves and S-waves, thereby achieving separation of the P-wave and S-wave. Although the separated $\mathrm{P}$-waves and S-waves given in their paper are scalar fields, the wave vector directions of the different waves in the Cartesian coordinate system have been calculated in the separation process, and according to the directions of the wave vectors, the scalar field could be restored to the vector field. Because this method can calculate the actual wave vector direction at every sample point, it provides a new approach for the de-noising technique based on the characteristics of wave vectors.

\section{Suggestion}

Great achievements have been obtained in vector field processing with the unremitting efforts of many scientific researchers $[30,32,35,83]$. However, generally speaking, the existing methods of multi-component seismic data vector processing are based on a relatively simple theoretical foundation, and so they can only process simple seismic signals. The processing capability is not sufficient for complex seismic signals modulated by complex inhomogeneous anisotropic media. 
The quaternion theory [84] and multilinear algebra theory [85] developed in recent years are very suitable for processing high-dimensional vector signals, and they have been successfully applied in the fields of electromagnetic physics [86], multi-channel digital communication [87], and color image processing $[88,89]$. Therefore, it will be of significant interest to study more sophisticated and systematic methods of multi-component seismic vector field processing based on the quaternion or multilinear algebra theory, thereby exploiting the potential capability of multi-component seismic data in solving the problem of complex anisotropic media.

\section{Conclusions and Perspectives}

The multi-component seismic wavefield is a physical vector field. To fully demonstrate the advantages of multi-component seismic data, we first have to retain the seismic vector field information during pre-processing, at least without interrupting the relative spatial direction and amplitude relationship between the multiple components. Secondly, we need an advanced technique to convert the kinematic and dynamic information hidden in the vector wavefield into the elastic parameters of the underground medium, such as velocity anisotropy, stress distribution, pore pressure, fluid saturation, and fracture size, which directly relates to oil and gas reservoirs. In this paper, we introduced several pre-processing methods for a multi-component seismic data vector field. The examples of real multi-component seismic data indicated that the vector processing technique could better avoid the distortion of vector field information and give more accurate predictions than the scalar processing technique.

Simply because studies on the extraction and utilization of seismic vector information are still in their infancy, vector processing techniques are currently outside of mainstream industrial interests. However, we believe this field will continue to develop, taking a key role in the future of fossil energy exploration, earthquake research, and micro-seismic monitoring.

Author Contributions: Conceptualization and formal analysis-Y.W. and P.S.; Investigation and methodology-C.W.; Reference collection-Y.L., The paper was written by all the authors.

Funding: This research was funded by the National Natural Science Foundation of China (Grant Nos. 41874166, 41504107, and U183208), the National Science and Technology Major Project (No. 2017ZX05018005), and the Research and Development Fund of Sinopec (No. P18070-5).

Conflicts of Interest: The authors declare no conflict of interest.

\section{References}

1. Mohammed, F.; Wang, J.Y. A review on multicomponent seismology: A potential seismic application for reservoir characterization. J. Adv. Res. 2016, 7, 515-524.

2. Barkved, O.; Bartman, B.; Gaiser, J.; Van Dok, R.; Johns, T.; Kristiansen, P.; Probert, T.; Thompson, M. The many facets of multicomponent seismic data. Oilfield Rev. 2004, 16, 42-56.

3. Crampin, S.; Peacock, S. A review of the current understanding of seismic shear-wave splitting in the Earth's crust and common fallacies in interpretation. Wave Motion 2008, 45, 675-722. [CrossRef]

4. Johnson, P.A.; Rasolofosaon, P.N.J. Nonlinear elasticity and stress-induced anisotropy in rock. J. Geophys. Res. Solid Earth 1996, 101, 3113-3124. [CrossRef]

5. Sun, Q.F.; Du, Q.Z. A review of the multi-component seismic data processing. Pet. Explor. Dev. 2011, 38, 67-73.

6. Tong, P.; Zhao, D.; Yang, D. Tomography of the 2011 Iwaki earthquake (M 7.0) and Fukushima nuclear power plant area. Solid Earth 2012, 3, 43-51. [CrossRef]

7. Wu, H.; Chen, J.; Huang, X.Y.; Yang, D.H. A New Earthquake Location Method Based on the Waveform Inversion. Commun. Comput. Phys. 2018, 23, 118-141. [CrossRef]

8. Crampin, S.; Gao, Y. A review of techniques for measuring shear-wave splitting above small earthquakes. Phys. Earth Planet. Inter. 2006, 159, 1-14. [CrossRef]

9. Zhang, Y.G.; Wang, Y.; Wang, M.Y. Some Key Problems in the Multi-Component Seismic Exploration. J. Geophys. 2004, 47, 151-155. 
10. Zhao, B.; Wang, Y.; Lu, J. New progress and key problems in multicomponent seismic exploration technology. Oil Geophys. Prospect. 2012, 47, 506-516.

11. Chen, H.; Yin, X.; Gao, J.; Liu, B.; Zhang, G. Seismic inversion for underground fractures detection based on effective anisotropy and fluid substitution. Sci. China Earth Sci. 2015, 58, 805-814. [CrossRef]

12. Yin, X.Y.; Zong, Z.Y.; Wu, G.C. Research on seismic fluid identification driven by rock physics. Sci. China Earth Sci. 2015, 45, 8-21. [CrossRef]

13. Lei, J. Impact of anisotropic and inhomogeneous medium on measuring seismic shear-wave splitting. Chin. Sci. Bull. 2017, 62, 2619-2629. [CrossRef]

14. Wang, Y.; Yang, D.H.; Yin, C.C.; Gao, Y. Anisotropic geophysics and vector field. Chin. Sci. Bull. 2017, 62, 2595-2605. [CrossRef]

15. An, S.P.; Hu, T.Y. Suppression of seismic surface waves based on adaptive weighted super-virtual interferometry. Sci. China Earth Sci. 2016, 46, 1371-1380. [CrossRef]

16. Li, Y.; Yang, B.; Lin, H.; Ma, H.; Nie, P. Suppression of strong random noise in seismic data by using time-frequency peak filtering. Sci. China Earth Sci. 2013, 43, 1123-1131. [CrossRef]

17. Zhao, B.L.; Shi, Y.M.; Yao, F.C.; Niu, Y.L.; Jiang, Y.; Chen, Z.D.; Wang, G.S.; Ma, X.Y. Prediction of the remaining oil distribution using multi-component seismic full waveform elastic inversion. Acta Pet. Sin. 2013, 34, 328-333.

18. Kamath, N.; Tsvankin, I. Elastic full-waveform inversion for VTI media: Methodology and sensitivity analysis. Geophysics 2016, 81, C53-C68. [CrossRef]

19. Zhang, Q.; McMechan, G.A. 2D and 3D elastic wavefield vector decomposition in the wavenumber domain for VTI media. Geophysics 2010, 75, D13-D26. [CrossRef]

20. Wang, W.L.; McMechan, G.A.; Zhang, Q.S. Comparison of two algorithms for isotropic elastic P and S vector decomposition. Geophysics 2015, 80, T147-T160. [CrossRef]

21. He, B.S.; Zhang, H.X. Vector prestack depth migration of multicomponent wavefield. Oil Geophys. Prospect. 2006, 41, 369-374.

22. Li, Z.C.; Yong, P.; Huang, J.P. Elastic wave reverse time migration based on vector wavefield seperation. J. China Univ. Pet. (Ed. Nat. Sci.) 2016, 40, 42-48.

23. Vidale, J.E. Complex polarization analysis of particle motion. Bull. Seismol. Soc. Am. 1986, 76, $1393-1405$.

24. Crampin, S.; Lovell, J.H. A decade of shear-wave splitting in the Earth's crust: What does it mean? What use can we make of it? And what should we do next? Geophys. J. R. Astron. Soc. 1991, 107, 387-407. [CrossRef]

25. Lilly, J.M.; Park, J. Multiwavelet spectral and polarization analyses of seismic records. Geophys. J. Int. 1995, 122, 1001-1021. [CrossRef]

26. Lu, J.; Wang, Y.; Yang, C.Y. Instantaneous polarization filtering focused on suppression of surface waves. Appl. Geophys. 2010, 7, 88-97. [CrossRef]

27. Reading, A.M.; Mao, W.; Gubbins, D. Polarization filtering for automatic picking of seismic data and improved converted phase detection. Geophys. J. Int. 2001, 147, 227-234. [CrossRef]

28. Diallo, M.S.; Kulesh, M.; Holschneider, M.; Scherbaum, F.; Adler, F. Characterization of polarization attributes of seismic waves using continuous wavelet transforms. Geophysics 2006, 71, V67-V77. [CrossRef]

29. Wang, C.; Wang, Y. Ground roll attenuation using polarization analysis in the t-f-k domain. Geophys. J. Int. 2017, 210, 240-254. [CrossRef]

30. Wang, C.; Wang, Y.; Wang, X.K.; Xun, C. Multicomponent seismic noise attenuation with multivariate order statistic filters. J. Appl. Geophys. 2016, 133, 70-81. [CrossRef]

31. Xun, C.; Wang, C.; Wang, Y. The application of multi-directional vector median filtering in multi-component seismic data. Geophys. Prospect. Pet. 2016, 55, 703-710.

32. Rodriguez, I.V.; Bonar, D.; Sacchi, M. Microseismic data denoising using a 3C group sparsity constrained time-frequency transform. Geophysics 2012, 77, V21-V29. [CrossRef]

33. Lei, J. A method for non-orthogonal seismic polarization-vector separation. Geophys. J. Int. 2005, 162, 965-974. [CrossRef]

34. Lu, J.; Wang, Y.; Yao, C. Separating P- and S-waves in an affine coordinate system. J. Geophys. Eng. 2012, 9, 12-18. [CrossRef]

35. Li, Z.Y. Separation of P-and S-Waves in Elastic Seismic Wavefield; University of Chinese Academy of Sciences: Beijing, China, 2013. 
36. Shimshoni, M.; Smith, S.W. Seismic signal enhancement with three-component detectors. Geophysics 1964, 29, 664-671. [CrossRef]

37. White, J.E. Motion product seismograms. Geophysics 1964, 29, 288-298. [CrossRef]

38. Chen, Y.; Gao, L.; Zhang, F.F. A method to enhance the signal/noise ratio of three component seismic data base on the polarization analysis in frequency domain. Prog. Geophys. 2007, 22, 255-261.

39. Du, Z.; Foulger, G.R.; Mao, W. Noise reduction for broad-band, three-component seismograms using data-adaptive polarization filters. Geophys. J. Int. 2000, 141, 820-828. [CrossRef]

40. Flinn, E.A. Signal analysis using rectilinearity and direction of particle motion. IEEE Proc. 1965, 12, 1874-1876. [CrossRef]

41. Jurkevics, A. Polarisation analyis of three-component array data. Bull. Seismol. Soc. Am. 1988, 78, 1725-1743.

42. Chen, H.F.; Li, X.Y.; Qian, Z.P.; Zhao, G.L. Robust adaptive polarization analysis method for eliminating ground roll in 3C land seismics. Appl. Geophys. 2013, 10, 295-304. [CrossRef]

43. Diallo, M.; Kulesh, M.; Holschneider, M. Instantaneous Polarization Attributes Based on Adaptive Covariance Method. Geophysics 2006, 71, V99. [CrossRef]

44. Ma, J.Q. Research on Adaptive Polarization Filtering for Multi-Component Seismic Data; Chang'an University: Chang'an, China, 2012.

45. René, R.M.; Fitter, J.L.; Forsyth, P.M.; Kim, K.Y.; Murray, D.J.; Walters, J.K.; Westerman, J.D. Multicomponent seismic studies using complex trace analysis. Geophysics 1986, 51, 1235-1251. [CrossRef]

46. Morozov, I.B.; Smithson, S.B. Instantaneous polarization attributes and directional fitering. Geophysics 1996, 61, 872-881. [CrossRef]

47. Schimmel, M.; Gallart, J. The use of instantaneous polarization attributes for seismic signal detection and image enhancement. Geophys. J. Int. 2003, 155, 653-668. [CrossRef]

48. Park, J.; Vernon, F.L.; Lindberg, C.R. Frequency Dependent Polarization Analysis of High-Frequency Seisrnograms. J. Geophys. Res. Solid Earth 1987, 92, 12664-12674. [CrossRef]

49. Kulesh, M.; Diallo, M.S.; Holschneider, M. Polarization analysis in the wavelet domain based on the adaptive covariance method. Geophys. J. Int. 2007, 170, 667-678. [CrossRef]

50. D'Auria, L.; Giudicepietro, F.; Martini, M. Polarization Analysis in the Discrete Wavelet Domain: An Application to Volcano Seismology. Bull. Seismol. Soc. Am. 2010, 100, 670-683. [CrossRef]

51. Pinnegar, C.R. Polarization analysis and polarization filtering of three-component signals with the time frequency S transform. Geophys. J. Int. 2006, 165, 596-606. [CrossRef]

52. Tan, Y.Y.; He, C.; Wang, Y.D.; Zhao, Z. Ground roll attenuation using a time-frequency dependent polarization filter based on the $\mathrm{S}$ transform. Appl. Geophys. 2013, 10, 279-294. [CrossRef]

53. Galiana-Merino, J.J.; Parolai, S.; Rosa-Herranz, J. Seismic wave characterization using complex trace analysis in the stationary wavelet packet domain. Soil Dyn. Earthq. Eng. 2011, 31, 1565-1578. [CrossRef]

54. Nikolaidis, N.; Pitas, I. Multichannel L filters based on reduced ordering. IEEE Trans. Circuits Syst. Video Technol. 1996, 6, 470-482. [CrossRef]

55. Pitas, I.; Tsakalides, P. Multivariate Ordering in Color Image Filtering. IEEE Trans. Circuits Syst. Video Technol. 1991, 1, 247-259. [CrossRef]

56. Lucat, L.; Siohan, P. Vector-median type filters and fast-computation algorithms. In Proceedings of the ISCAS '97-IEEE International Symposium on Circuits and Systems, Hong Kong, China, 9-12 June 1997; Volumes I-IV, pp. 2469-2472.

57. Xu, J.T.; Wang, L.; Shi, Z.F. A switching weighted vector median filter based on edge detection. Signal Process. 2014, 98, 359-369. [CrossRef]

58. Trahanias, P.E.; Karakos, D.; Venetsanopoulos, A.N. Directional processing of color images: Theory and experimental results. IEEE Trans. Image Process. 1996, 5, 868-880. [CrossRef]

59. Pitas, I.; Venetsanopoulos, A.N. Order-Statistics in Digital Image-Processing. Proc. IEEE 1992, 80, $1893-1921$. [CrossRef]

60. Huo, S.D.; Luo, Y.; Kelamis, P.G. Simultaneous sources separation via multidirectional vector-median filtering. Geophysics 2012, 77, V123-V131. [CrossRef]

61. Liu, Y.K. Noise reduction by vector median filtering. Geophysics 2013, 78, V79-V86. [CrossRef] 
62. Aharon, M. Overcomplete Dictionaries for Sparse Representation of Signals; Technion-Israel Institute of Technology: Haifa, Israel, 2006.

63. Aharon, M.; Elad, M.; Bruckstein, A. K-SVD: An algorithm for designing overcomplete dictionaries for sparse representation. IEEE Trans. Signal Process. 2006, 54, 4311-4322. [CrossRef]

64. Mallat, S.G.; Zhang, Z.F. Matching Pursuits with Time-Frequency Dictionaries. IEEE Trans. Signal Process. 1993, 41, 3397-3415. [CrossRef]

65. Tropp, J.A. Greed is good: Algorithmic results for sparse approximation. IEEE Trans. Inf. Theory 2004, 50, 2231-2242. [CrossRef]

66. Chen, S.S.B.; Donoho, D.L.; Saunders, M.A. Atomic decomposition by basis pursuit. Siam Rev. 2001, 43, 129-159. [CrossRef]

67. Li, H.S.; Wu, G.C.; Yin, X.Y. Morphological component analysis in seismic data reconstrution. Oil Geophys. Prospect. 2012, 47, 236-243.

68. Chen, W.C.; Wang, W.; Gao, J.H. Sparsity optimized separation of Ground-roll noise based on morphological diversity of seismic waveform components. Chin. J. Geophys. 2013, 56, 2771-2782.

69. Xu, X.H.; Qu, G.Z.; Zhang, Y.; Bi, Y.Y.; Wang, J.J. Ground-roll separation of seismic data based on morphological component analysis in two-dimensional domain. Appl. Geophys. 2016, 13, 116-126. [CrossRef]

70. Liang, D.H.; Chen, S.C. Deconvolution of seismic data based on L0 norm sparse constrain. Geophys. Prospect. Pet. 2014, 53, 397-403.

71. Yuan, M.; Lin, Y. Model selection and estimation in regression with grouped variables. J. R. Stat. Soc. Ser. $B$ Stat. Methodol. 2006, 68, 49-67. [CrossRef]

72. Fornasier, M.; Rauhut, H. Recovery algorithms for vector-valued data with joint sparsity constraints. Siam J. Numer. Anal. 2008, 46, 577-613. [CrossRef]

73. Eldar, Y.C.; Bolcskei, H. Block-sparsity: Coherence and efficient recovery. In Proceedings of the IEEE International Conference on Acoustics, Speech, and Signal Processing, Taipei, Taiwan, 19-24 April 2009; pp. 2885-2888.

74. Beck, A.; Teboulle, M. A Fast Iterative Shrinkage-Thresholding Algorithm for Linear Inverse Problems. Siam J. Imaging Sci. 2009, 2, 183-202. [CrossRef]

75. Jiang, X.X.; Lin, J.; Ye, F.; Zheng, F. Separation of P-P and P-SV wavefields by high resolution parabolic Radon transform. J. Appl. Geophys. 2015, 119, 192-201. [CrossRef]

76. Hu, T.Y.; Zhang, G.J.; Zhao, W.; Wen, S.L. Decompostion of multicomponent seismic wavefields. Chin. J. Geophys. 2004, 47, 504-508. [CrossRef]

77. Yao, D.Z.; Zhou, X.X.; Zhong, B.S. Method for separating out P-wave or S-wave in VSP data, and its application. Oil Geophys. Prospect. 1993, 28, 623-628.

78. Sun, R.; McMechan, G.A.; Hsiao, H.H.; Chow, J. Separating P- and S-waves in prestack 3D elastic seismograms using divergence and curl. Geophysics 2004, 69, 286-297. [CrossRef]

79. Yan, J.; Sava, P. Elastic wavefield separation for VTI media. In Proceedings of the 78th Annual International Meeting, Columbus, OH, USA, 24-29 October 2008; pp. 2191-2195.

80. Li, Z.Y.; Gu, B.L.; Ma, X.N.; Liang, G.H. Separating P- and S-waves in prestack elastic seismograms using the equivalent form of elastic wave equation. J. Appl. Geophys. 2015, 114, 210-223. [CrossRef]

81. Ma, D.T.; Zhu, G.M. Numerical modeling of P-wave and S-wave separation in elastic wavefield. Oil Geophys. Prospect. 2003, 38, 482-486.

82. Li, X.Y.; MacBeth, C.; Crampin, S. Interpreting non-orthogonal split shear waves for seismic anisotropy in multicomponent VSPS. Geophys. Prospect. 1998, 46, 1-27. [CrossRef]

83. Song, Z.C.; Lei, J. Analyzing and correcting the scatter in measurement of time-delays between fast ans slow shear-waves with near-field earthquakes. Chin. Sci. Bull. 2017, 62, 3356-3368. [CrossRef]

84. Hitzer, E.; Sangwine, S.J. Quaternion and Clifford Fourier Transforms and Wavelets; Birkhäuser: Basel, Switzerland, 2015.

85. De Lathauwer, L. Signal Processing Based on Multilinear Algebra. Ph.D. Thesis, Katholieke Universiteit, Leuven, Belgium, 1997.

86. Esch, J. Geometric Algebra for Electrical and Electronic Engineers. Proc. IEEE 2014, 102, 1338-1339. [CrossRef]

87. Paulus, C.; Mars, J.; Gounon, P. Wideband spectral matrix filtering for multicomponent sensors array. Signal Process. 2005, 85, 1723-1743. [CrossRef] 
88. Chan, W.L.; Choi, H.; Baraniuk, R.G. Coherent multiscale image processing using dual-tree quaternion wavelets. IEEE Trans. Image Process. 2008, 17, 1069-1082. [CrossRef]

89. Jia, X.N. Quaternion Bispectrum and Its Application in Color Image Processiong; Jilin University: Changchun, China, 2014. 